\title{
Genesis and evolution of dust in galaxies in the early Universe
}

\section{Rapid dust evolution in quasars at $z \gtrsim 6$}

\author{
C. Gall, A. C. Andersen, and J. Hjorth \\ Dark Cosmology Centre, University of Copenhagen, Niels Bohr Institute, Juliane Maries Vej 30, 2100 Copenhagen, Denmark
} e-mail: christa@dark-cosmology.dk

Received 18 August 2010 / Accepted 7 January 2011

\begin{abstract}
Aims. We intend to assess the most plausible scenarios for generating large amounts of dust in high- $z$ quasars (QSOs) on the basis of observationally derived physical properties of QSOs at $z \gtrsim 6$.

Methods. We use a chemical evolution model to compute the temporal progression of quantities such as the amount of dust and gas, stellar masses, star formation rates (SFRs) and the metallicity for various combinations of the initial mass function (IMF), the mass of the galaxy, dust production efficiencies, and the degree of dust destruction in the ISM. We investigate the influence of the SFR on the evolution of these quantities, and determine the earliest epochs at which agreement with observations can be achieved. We apply the obtained results to individual QSOs at $z \gtrsim 6$.

Results. We find that large quantities of dust can be generated rapidly as early as $30 \mathrm{Myr}$ after the onset of the starburst when the SFR of the starburst is $\gtrsim 10^{3} M_{\odot} \mathrm{yr}^{-1}$. The amount of dust and several other physical quantities of individual QSOs at $z \gtrsim 6$ are satisfactorily reproduced by models at epochs $30,70,100$, and $170 \mathrm{Myr}$ for galaxies with initial gas masses of $1-3 \times 10^{11} M_{\odot}$. The best agreement with observations is obtained with top-heavy IMFs. A sizable dust contribution from supernovae (SNe) is however required, while at these epochs dust production by asymptotic giant branch (AGB) stars is negligible. Moderate dust destruction in the ISM can be accommodated.
\end{abstract}

Key words. galaxies: high-redshift - galaxies: starburst - galaxies: evolution - evolution - quasars: general - stars: massive

\section{Introduction}

Studying QSOs and their host galaxies at high redshift $(z>6)$ is important to gain deeper insight into the formation and evolution of galaxies, the origin of dust production, and the build up of stellar bulge masses in coevolution with supermassive black holes (SMBHs).

While the most distant known QSO, J114816.64+525150.3 (Fan et al. 2003, herafter J1148+5251), is at $z=6.4$, several tens of QSOs have been discovered at $z \sim 6$ (e.g., Fan et al. 2004, 2006; Willott et al. 2007; Jiang et al. 2010). Most of the observed QSOs at this redshift, where the epoch of cosmic evolution is $\sim 1 \mathrm{Gyr}$, exhibit extreme physical properties such as very high far-infrared (FIR) luminosities which imply large dust masses (e.g., Omont et al. 2001, 2003; Carilli et al. 2001; Bertoldi \& Cox 2002), and SMBHs with masses $>10^{9} M_{\odot}$ (e.g., Barth et al. 2003; Willott et al. 2003; Vestergaard 2004).

Observations of QSOs have shown that dust emission at near-infrared (NIR) wavelengths arise from warm and hot dust $(T \lesssim 1000 \mathrm{~K}$ ) assembled within a few parsec (e.g., Hines et al. 2006; Jiang et al. 2006). The NIR emission is believed to be powered by the active galactic nucleus and related to the QSO activity (e.g., Polletta et al. 2000). However, two QSOs at $z \sim 6$ without detectable emission from hot dust have been found (Jiang et al. 2006, 2010). It has been proposed that these QSOs are at a too early evolutionary stage to have built up significant amounts of hot dust. Alternative scenarios including for example the destruction of the hot dust or dust misalignments from the SMBH have also been discussed (Hao et al. 2010a,b; Guedes et al. 2010).

The FIR luminosity of $L_{\mathrm{FIR}} \sim 10^{12-13} L_{\odot}$ is attributed to cold dust ( $T \sim 30-60 \mathrm{~K})$ (e.g., Wang et al. 2008) which is probably distributed over kilo-parsec scales throughout the host galaxy (Leipski et al. 2010). The amount of cold dust inferred is about a few times $10^{8} M_{\odot}$ (e.g., Bertoldi et al. 2003a; Robson et al. 2004; Beelen et al. 2006; Michałowski et al. 2010). The dominant source of the high FIR luminosity is believed to be dust heated by intense star formation in the circumnuclear region (e.g., Carilli et al. 2004; Riechers et al. 2007; Wang et al. 2008). Detection of [ $\mathrm{C}_{\mathrm{II}}$ ] line emission at $158 \mu \mathrm{m}$ (Maiolino et al. 2005) within a central region with radius $\sim 750 \mathrm{pc}$ of the host galaxy of $\mathrm{J} 1148+5251$ also implies a high star formation rate surface density of $1000 M_{\odot} \mathrm{yr}^{-1} \mathrm{kpc}^{-2}$ (Walter et al. 2009). Wang et al. (2010) derived SFRs between 530-2300 $M_{\odot} \mathrm{yr}^{-1}$ from observations of a sample of QSOs at redshift $z>5$. Observations of strong metal emission of high-z QSOs (e.g., Barth et al. 2003; Dietrich et al. 2003; Maiolino et al. 2003; Becker et al. 2006) indicate strong star forming activity in the QSO hosts and solar or supersolar metallicity (e.g., Fan et al. 2003; Freudling et al. 2003; Juarez et al. 2009). Theoretical studies of the gas metallicity of QSO hosts also predict supersolar metallicities for $z=5-6$ QSOs (e.g., Di Matteo et al. 2004).

The high inferred SFRs imply short timescales $\left(\leq 10^{8} \mathrm{yr}\right)$ of the starburst (e.g. Bertoldi et al. 2003a; Walter et al. 2004; Dwek et al. 2007; Riechers et al. 2009), and consequently a young age of the QSOs. An early evolutionary stage of $z>4$ QSOs has 
also been suggested from studies of extinction curves of broad absorption line QSOs (e.g., Gallerani et al. 2010) which turned out to be best fitted with extinction curves for SN-like dust (e.g., Maiolino et al. 2004, 2006; Gallerani et al. 2010). This suggests $\mathrm{SNe}$ as the preferential source of dust at early epochs (e.g., Dwek 1998; Morgan \& Edmunds 2003; Hirashita et al. 2005; Dwek et al. 2007; Dwek \& Cherchneff 2011), even though the dust productivity of $\mathrm{SNe}$ is poorly constrained (for a review see Gall et al. in prep). The dust in high-z QSOs could also be grown in the ISM (e.g., Draine 2009; Michałowski et al. 2010; Pipino et al. 2011). Finally, a dominant dust production by asymptotic giant branch stars has been claimed (Valiante et al. 2009).

Molecular gas masses of the order of $\sim 1-2.5 \times 10^{10} M_{\odot}$ have been inferred from detections of high excitation CO line emission in QSOs at $z>5$ within a $\sim 2.5 \mathrm{kpc}$ radius region (e.g., Bertoldi et al. 2003b; Walter et al. 2003, 2004; Wang et al. 2010). The dynamical masses inferred from these $\mathrm{CO}$ observations are a few times $\sim 10^{10-11} M_{\odot}$ which sets an upper limit on stellar bulge masses. These however are roughly two orders of magnitude lower than required from the present day black hole-bulge relation (e.g., Marconi \& Hunt 2003). It therefore has been proposed that the formation of the SMBH occurs prior to the formation of the stellar bulge. QSOs will then have to accrete additional material to build up the required bulge mass (e.g., Walter et al. 2004; Riechers et al. 2009; Wang et al. 2010). For QSOs at $z>6$ superEddington growth on timescales shorter than $\sim 10^{8}$ yr seem to be required to form a $\mathrm{SMBH}>10^{9} M_{\odot}$ (e.g., Kawakatu \& Wada 2009). It has also been predicted that QSOs at $z \sim 6$ likely have formed in dark matter halos of $10^{12-13} M_{\odot}$ (e.g., Li et al. 2007; Kawakatu \& Wada 2009).

In Gall et al. (2011, herafter Paper I) we developed a chemical evolution model to elucidate the conditions required for generating large dust masses in high- $z$ starburst galaxies. We showed that galaxies with masses of $1-5 \times 10^{11} M_{\odot}$ are suitable for enabling the production of large amounts of dust within $\sim 400 \mathrm{Myr}$. In the present paper we apply this model to QSOs at $z \gtrsim 6$. We perform more detailed comparison between model results and values inferred from observations of $z \gtrsim 6$ QSOs to identify the most likely scenario. Furthermore, we consult additional parameters such as the $\mathrm{H}_{2}$ mass and the $\mathrm{CO}$ conversion factor for more refined evaluations. In particular, calculations with higher SFRs than in Paper I are considered. We aim to determine the earliest epochs at which the model results are in agreement with those from observations.

The structure of the paper is as follows: in Sect. 2 we briefly review the model developed in Paper I. A detailed analysis of the results is presented in Sect. 3 followed by a discussion in Sect. 4.

\section{The model}

The galactic chemical evolution model from Paper I is selfconsistent, numerically solved and has been developed to ascertain the temporal progression of dust, gas, metals, and diverse physical properties of starburst galaxies. The incorporated stellar sources are AGB stars in the mass range 3-8 $M_{\odot}$ and $\mathrm{SNe}$. A differentiation between diverse $\mathrm{SN}$ subtypes has been implemented. Their roles as sources of dust production, dust destruction or suppliers of gas and heavy elements are taken into account. The lifetime dependent yield injection by the stellar sources, as well as dust destruction in the ISM due to SN shocks are also taken into account. Moreover, the formation of a SMBH is considered. Due to the very high SFRs of the starbursts, infall of neutral gas will only effect the system for comparable high infall rates. Thus, gas infall and outflows are neglected. Possible
Table 1. Model parameters.

\begin{tabular}{llll}
\hline \hline Parameters & Value & Unit & Description \\
\hline$\psi_{\text {ini }}$ & $3 \times 10^{3}, 1 \times 10^{4}$ & $M_{\odot} \mathrm{yr}^{-1}$ & Star formation rate \\
$M_{\text {SMBH }}$ & $3 \times 10^{9}$ & $M_{\odot}$ & Mass of the SMBH \\
$t_{\text {SMBH }}$ & $1 \times 10^{8}$ & $\mathrm{yr}$ & $\begin{array}{l}\text { Growth timscale } \\
\text { for the SMBH }\end{array}$ \\
\hline
\end{tabular}

caveats of such an approach are discussed in Paper I. The model allows investigations of a broad range of physical properties of galaxies.

The prime parameters are summarized in the following.

- Three different possible prescriptions for the stellar yields of SNe are implemented, i.e., (i) stellar evolution models by Eldridge et al. (2008) (referred to as "EIT08M"); (ii) rotating stellar models by Georgy et al. (2009); or (iii) nucleosynthesis models by either Woosley \& Weaver (1995) or Nomoto et al. (2006). The stellar yields for AGB stars are taken from van den Hoek \& Groenewegen (1997).

- We differentiate between five different IMFs. These are a Salpeter (1955) IMF, a top-heavy, and a mass-heavy IMF, as well as IMFs (Larson 1998) with characteristic masses of either $m_{\mathrm{ch}}=0.35$ (Larson 1$)$ or $m_{\mathrm{ch}}=10$ (Larson 2).

- The SFR at a certain epoch is given by the Kennicutt law (Kennicutt 1998) as $\psi(t)=\psi_{\text {ini }}\left(M_{\mathrm{ISM}}(t) / M_{\text {ini }}\right)^{k}$, where $\psi_{\text {ini }}$ is the initial SFR of the starburst, $M_{\mathrm{ISM}}(t)$ is the initial gas mass of the galaxy and $k=1.5$.

- The amount of dust produced by SNe and AGB stars is calculated using the dust formation efficiencies discussed in Paper I. For SNe three different dust production efficiency limits are determined, i.e. a "maximum" $\mathrm{SN}$ efficiency, a "high" SN efficiency, and a "low" SN efficiency. The "maximum" SN efficiency originates from theoretical SN dust formation models, and corresponds to dust masses of approximately $3-10 \times 10^{-1} M_{\odot}$. Similar dust masses have been observed in SN remnants such as Cas A (e.g., Dunne et al. 2009) or Kepler (e.g., Gomez et al. 2009). Dust destruction in reverse shock interaction of about $93 \%$ has been applied to the "maximum" SN efficiency, to obtain the "high" SN efficiency. The amount of dust for instance is $\sim 2-6 \times 10^{-2} M_{\odot}$, which is also comparable to some observations of older SN remnants (Paper I, see references therein). The "low" SN efficiency is based on SN dust yields (on average about $3 \times 10^{-3} M_{\odot}$ ) inferred from observations of SN ejecta.

- Dust destruction in the ISM is implemented in terms of the mass of ISM material, $M_{\mathrm{cl}}$, swept up by a single SN shock and cleared of the containing dust.

For calculations in this paper most parameters have the same settings as defined in Paper I. We apply the models where the formation of a SMBH has been included. A constant growth rate has been estimated based on the final mass of the SMBH and the considered growth timescale. In this paper the SMBH growth is considered with a shorter growth timescale and calculations are performed with higher initial SFRs. For the SN yields we only consider the case of EIT08M. The parameters which differ from those used in Paper I are listed in Table 1.

\section{Results}

In this section we present the results of models calculated within short timescales after the starburst. 
C. Gall et al.: Genesis and evolution of dust in galaxies in the early Universe. II.

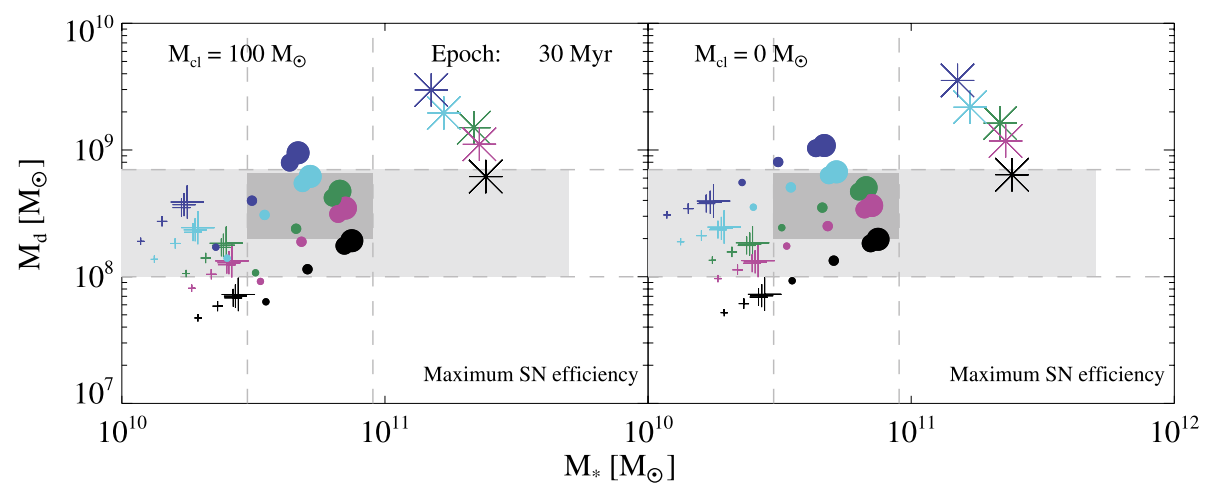

Fig. 1. Relation between dust mass and stellar mass at an epoch of $30 \mathrm{Myr}$, for various initial gas masses and IMFs. Calculations are performed for a "maximum" SN efficiency and dust destruction in the ISM with $M_{\mathrm{cl}}=100 M_{\odot}$ (left panel) and $M_{\mathrm{cl}}=0$ (right panel). The colored symbols are obtained for different initial gas masses, $M_{\text {ini }}$, SFRs, and IMFs. The size of the symbols is scaled by $M_{\text {ini }}$. Calculations are made for $M_{\text {ini }}=$ $1.3 \times 10^{12} M_{\odot}$ (largest symbol), $M_{\text {ini }}=5 \times 10^{11} M_{\odot}, M_{\text {ini }}=3 \times 10^{11} M_{\odot}, M_{\text {ini }}=1 \times 10^{11} M_{\odot}$, and $M_{\text {ini }}=5 \times 10^{10} M_{\odot}$ (smallest symbol). The crosses correspond to calculations for a initial SFR $\psi_{\mathrm{ini}}=10^{3} M_{\odot} \mathrm{yr}^{-1}$, the filled circles to $\psi_{\mathrm{ini}}=3 \times 10^{3} M_{\odot} \mathrm{yr}^{-1}$, and the stars to $\psi_{\mathrm{ini}}=10^{4} M_{\odot} \mathrm{yr}^{-1}$. The black, green, cyan, magenta, and blue colors denote the Salpeter, mass-heavy, top-heavy, Larson 1, and Larson 2 IMF, respectively. The dark grey region indicates the mass range of stellar masses and dust masses derived from observations of QSOs at $z>6$. The vertical dashed lines represent the lower and upper limits of the observed stellar masses. The light grey area illustrates the whole mass ranges derived from observations of QSOs $>5$ and accounts for uncertainties in the derived quantities. The horizontal dashed lines mark the lower and upper mass limit of the derived dust masses.

A short enrichment timescale of a few times $10^{7} \mathrm{yr}$ for an intense starburst with a SFR of $\sim 3 \times 10^{3} M_{\odot} \mathrm{yr}^{-1}$ has been proposed by e.g., Bertoldi et al. (2003a), Walter et al. (2004), Dwek et al. (2007), Riechers et al. (2009). Owing to this suggestion we are interested in whether the observed large dust masses in excess of $10^{8} M_{\odot}$ can be reached within about $100 \mathrm{Myr}$. Consequently we performed calculations with an initial SFR for the starburst with $\psi_{\text {ini }}=3 \times 10^{3} M_{\odot} \mathrm{yr}^{-1}$ for galaxies with initial gas masses $M_{\text {ini }}=5 \times 10^{10} M_{\odot}, M_{\text {ini }}=1 \times 10^{11} M_{\odot}, M_{\text {ini }}=3 \times 10^{11} M_{\odot}$, and $M_{\text {ini }}=5 \times 10^{11} M_{\odot}$. For the most massive system with $M_{\text {ini }}=1.3 \times 10^{12} M_{\odot}$ an initial SFR $\psi_{\text {ini }}=1 \times 10^{4} M_{\odot} \mathrm{yr}^{-1}$ is adopted. We included the results for a lower initial SFR of $10^{3} M_{\odot} \mathrm{yr}^{-1}$ from models computed in Paper I for comparison.

In Paper I we analyzed the evolution of the amount of dust and various physical properties, and found that these are strongly dependent on the mass of the galaxy. Moreover, for a given initial SFR all quantities evolve faster in less massive galaxies. In this paper we perform detailed comparisons between calculated and observed values of the total dust mass, $M_{\mathrm{d}}$, the stellar mass, $M_{*}$, the SFR, $\psi$, and the metallicity, $Z$. We identified the shortest epoch, where some model results are in accordance with observations to be $30 \mathrm{Myr}$. Furthermore, we discuss quantities such as the $\mathrm{CO}$ conversion factor, the gas-to- $\mathrm{H}_{2}$ mass ratio, and the possible amount of molecular hydrogen.

\subsection{Dust and stellar mass}

In Fig. 1 we present the results for the mass of dust versus the stellar mass for galaxies with different initial gas masses and initial SFRs at an epoch of $30 \mathrm{Myr}$. The displayed models are computed for a "maximum" SN efficiency. Dust destruction in the ISM is considered for values of $M_{\mathrm{cl}}=100 M_{\odot}$ (left panel) and $M_{\mathrm{cl}}=0$ (right panel).

The dark grey region represents the mass ranges of the stellar mass and dust mass derived from observations of QSOs at $z>6$. The lower and upper limits of the stellar mass are estimated by subtracting the molecular gas masses, $M_{\mathrm{H}_{2}}$ from the total dynamical masses, $M_{\text {dyn }}$. Values for $M_{\text {dyn }}$ and $M_{\mathrm{H}_{2}}$ are based on data from Wang et al. (2010, and references therein) for three QSOs at $z>6$. For an estimation of $M_{\mathrm{dyn}}$ an inclination angle $i=65^{\circ}$ of the gas disk is taken for QSO J1148+5251 (Walter et al. 2004), while $i=40^{\circ}$ similar to Wang et al. (2010) is applied to the remaining two QSOs. We adopt the lower and upper limits for the dust masses from Beelen et al. (2006) and Michałowski et al. (2010). The light grey region covers the range of derived stellar masses and dust masses from observations of QSOs > 5 (Wang et al. 2010; Michałowski et al. 2010). The boundaries for the stellar masses are estimated similar to the QSOs at $z>6$ (with $i=40^{\circ}$ for deriving $M_{\mathrm{dyn}}$ ). We set the lower dust limit to $10^{8} M_{\odot}$ to account for the uncertainties of derived dust masses from observations.

Despite the short time span of $30 \mathrm{Myr}$, it is evident that most models are within the plausible mass ranges illustrated by the light and dark grey regions. This signifies a rapid build-up of a large amount of dust, provided SNe produced dust with a "maximum" SN efficiency. For galaxies with $M_{\text {ini }}=1-5 \times 10^{11} M_{\odot}$ all models with an initial SFR of $3 \times 10^{3} M_{\odot} \mathrm{yr}^{-1}$ are in agreement with the observed values for the stellar masses for QSOs at $z>6$. The requirements for $M_{\mathrm{d}}$ are best accomplished with either a top-heavy, mass-heavy or Larson 1 IMF for both values of $M_{\mathrm{cl}}$. In a galaxy with $M_{\mathrm{ini}}=1 \times 10^{11} M_{\odot}$ the amount of dust reached with a Larson $2 \mathrm{IMF}$ and $M_{\mathrm{cl}}=100 M_{\odot}$ also matches with the dark grey region. Models for either a "high" or "low" SN efficiency did not reach $10^{8} M_{\odot}$ of dust. Only in the most massive galaxy $\left(M_{\text {ini }}=1.3 \times 10^{12} M_{\odot}\right)$ and for top-heavy IMFs with a 'high' SN efficiency an amount of dust $>10^{8} M_{\odot}$ is obtained.

In Fig. 2 we illustrate the results for dust and stellar masses at an epoch of 100 Myr. We present models for a "maximum" SN efficiency (top row) and a "high" SN efficiency (bottom row), while dust destruction in the ISM is considered for a $M_{\mathrm{cl}}=800 M_{\odot}$ (left column), $M_{\mathrm{cl}}=100 M_{\odot}$ (middle column), and $M_{\mathrm{cl}}=0$ (right column). We carried out calculations for a "low" SN efficiency, but the obtained dust masses of these models remained below $10^{8} M_{\odot}$.

At these early epochs the stellar mass, $M_{*}$, is higher for models with an initially larger SFR (at fixed IMF and $M_{\text {ini }}$ ). The stellar mass is also larger for IMFs biased towards low mass stars (at fixed $M_{\text {ini }}$ and $\psi_{\text {ini }}$ ). It is interesting to note that in the less massive galaxies $\left(0.5-1 \times 10^{11} M_{\odot}\right)$ dust masses obtained 


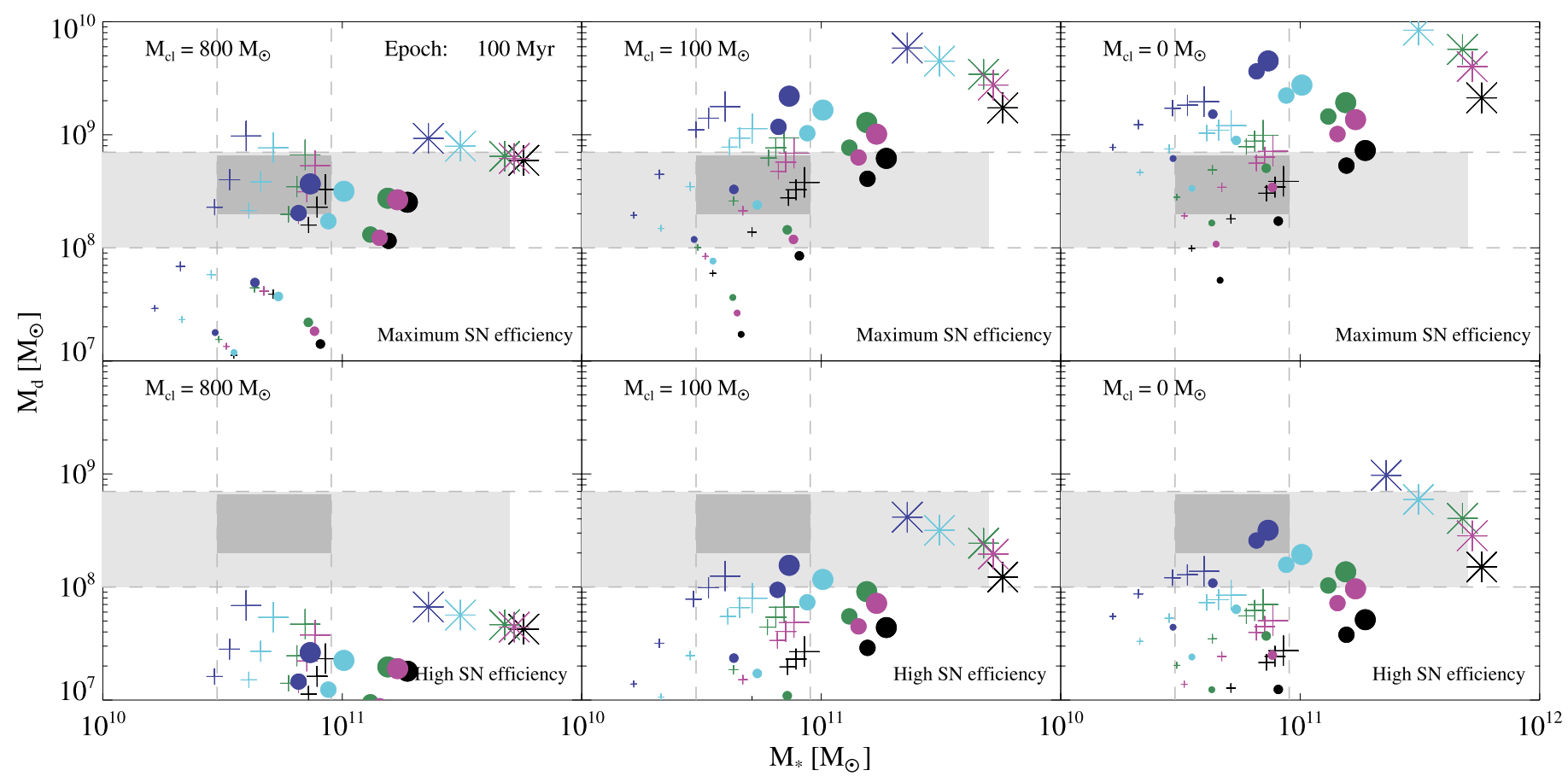

Fig. 2. Relation between dust mass and stellar mass at an epoch of $100 \mathrm{Myr}$ for various initial gas masses and IMFs. Calculations are performed for a "maximum" SN efficiency (top row) and a "high" SN efficiency (bottom row). Dust destruction in the ISM is considered for a $M_{\mathrm{cl}}=800 M_{\odot}$ (left column), $M_{\mathrm{cl}}=100 M_{\odot}$ (middle column), and $M_{\mathrm{cl}}=0$ (right column). The colored symbols are obtained for different initial gas masses, $M_{\mathrm{ini}}$, SFRs, and IMFs. The size of the symbols is scaled by $M_{\text {ini }}$. Calculations are made for $M_{\text {ini }}=1.3 \times 10^{12} M_{\odot}\left(\right.$ largest symbol), $M_{\text {ini }}=5 \times 10^{11} M_{\odot}$, $M_{\text {ini }}=3 \times 10^{11} M_{\odot}, M_{\text {ini }}=1 \times 10^{11} M_{\odot}$ and $M_{\text {ini }}=5 \times 10^{10} M_{\odot}$ (smallest symbol). The crosses correspond to calculations for a initial SFR $\psi_{\mathrm{ini}}=10^{3} M_{\odot} \mathrm{yr}^{-1}$, the filled circles to $\psi_{\mathrm{ini}}=3 \times 10^{3} M_{\odot} \mathrm{yr}^{-1}$, and the stars to $\psi_{\text {ini }}=10^{4} M_{\odot} \mathrm{yr}^{-1}$. The black, green, cyan, magenta, and blue colors denote the Salpeter, mass-heavy, top-heavy, Larson 1, and Larson 2 IMF, respectively. The dark grey region indicates the mass range of stellar masses and dust masses derived from observations of QSOs at $z>6$. The vertical dashed lines represent the lower and upper limits of the observed stellar masses. The light grey area illustrates the whole mass ranges derived from observations of QSOs $>5$ and accounts for uncertainties in the derived quantities. The horizontal dashed lines mark the lower and upper mass limit of the derived dust masses.

for the higher initial SFR $\left(\psi_{\text {ini }}=3 \times 10^{3} M_{\odot} \mathrm{yr}^{-1}\right)$ are lower than dust masses obtained for the lower SFR $\left(\psi_{\text {ini }}=10^{3} M_{\odot} \mathrm{yr}^{-1}\right)$. Moreover, in these galaxies the amount of dust reached at an epoch of $30 \mathrm{Myr}$ (see Fig. 1) and for $M_{\mathrm{cl}}=100-800 M_{\odot}$ is also higher than that seen at the epoch of $100 \mathrm{Myr}$ for same $M_{\mathrm{cl}}$.

We find that the stellar masses for models with an initial SFR $\psi_{\text {ini }}=1-3 \times 10^{3} M_{\odot} \mathrm{yr}^{-1}$ are within the observed region for $z>5$ QSOs. For some models with $\psi_{\text {ini }}=3 \times 10^{3} M_{\odot} \mathrm{yr}^{-1}$, stellar masses are within the mass range for $z>6$ QSOs. This in particular applies to systems with either $M_{\text {ini }}=0.5-$ $1 \times 10^{11} M_{\odot}($ all IMFs $)$ or $M_{\text {ini }}=3-5 \times 10^{11} M_{\odot}$ with top heavy IMFs. Stellar masses within the dark grey area are also found with $\psi_{\text {ini }}=10^{3} M_{\odot} \mathrm{yr}^{-1}$ for galaxies with either $M_{\text {ini }}=3-$ $13 \times 10^{11} M_{\odot}$ and top heavy IMFs or for the less massive galaxies in combination with IMFs favoring low mass stars.

In the case of $M_{\mathrm{cl}}=800 M_{\odot}$ and for a "maximum" SN efficiency most models with $M_{\text {ini }}=3-13 \times 10^{11} M_{\odot}$ and $\psi_{\text {ini }}=10^{3} M_{\odot} \mathrm{yr}^{-1}$ fit within the dark grey region. However for the higher initial SFR $M_{\mathrm{d}}$ is within or close to this zone only for galaxies with $M_{\text {ini }}=3-5 \times 10^{11} M_{\odot}$ and top-heavy IMFs. For $M_{\mathrm{cl}}=100 M_{\odot}$ and a "maximum" SN efficiency the dust mass obtained in a galaxy with $M_{\text {ini }}=1 \times 10^{11} M_{\odot}$, $\psi_{\text {ini }}=3 \times 10^{3} M_{\odot} \mathrm{yr}^{-1}$ and for top-heavy IMFs is in agreement with observations, while the dust masses in the more massive galaxies for some IMFs and SFRs are higher than required. In the case of no dust destruction the dust masses reached for some IMFs and SFRs are able to match within the dark grey area also in the least massive galaxy.
We find that in case of a "high" SN efficiency and for $\psi_{\text {ini }}=$ $3 \times 10^{3} M_{\odot} \mathrm{yr}^{-1}$ in galaxies with initial masses $3-5 \times 10^{11} M_{\odot}$ and top-heavy IMFs high dust masses are possible, even if dust destruction is included (i.e., $M_{\mathrm{cl}}=0-100 M_{\odot}$ ).

\subsection{Metallicity and SFR}

We next present the obtained metallicities and SFRs at the time of observation for the models discussed above.

Figure 3 depicts the metallicity versus SFR at epochs of $30 \mathrm{Myr}$ (left panel) and $100 \mathrm{Myr}$ (right panel). With respect to observations of QSOs > (5) 6 we marked the range of derived values as a dark grey shaded zone. The lower and upper limits of the SFR are based on observations by Bertoldi et al. (2003a) and Wang et al. (2010). We set the lower limit for the metallicity at the solar value and the upper limit at $5 Z_{\odot}$. This is based on the inferred solar or supersolar metallicities in high- $z$ QSOs (e.g., Barth et al. 2003; Dietrich et al. 2003; Fan et al. 2003; Freudling et al. 2003; Maiolino et al. 2003; Di Matteo et al. 2004; Becker et al. 2006; Juarez et al. 2009). We note that there are no strong constraints on the upper limit and therefore the zone above $5 Z_{\odot}$ is marked as light grey shaded region to account for the uncertainty.

We find that at an epoch of 30 Myr high metallicities in the less massive galaxies are already reached. The best result is attained by a system with $M_{\text {ini }}=1 \times 10^{11} M_{\odot}, \psi_{\text {ini }}=$ $3 \times 10^{3} M_{\odot} \mathrm{yr}^{-1}$, and IMFs biased towards higher masses. For a galaxy with $M_{\text {ini }}=5 \times 10^{10} M_{\odot}$ all models with either the 
C. Gall et al.: Genesis and evolution of dust in galaxies in the early Universe. II.

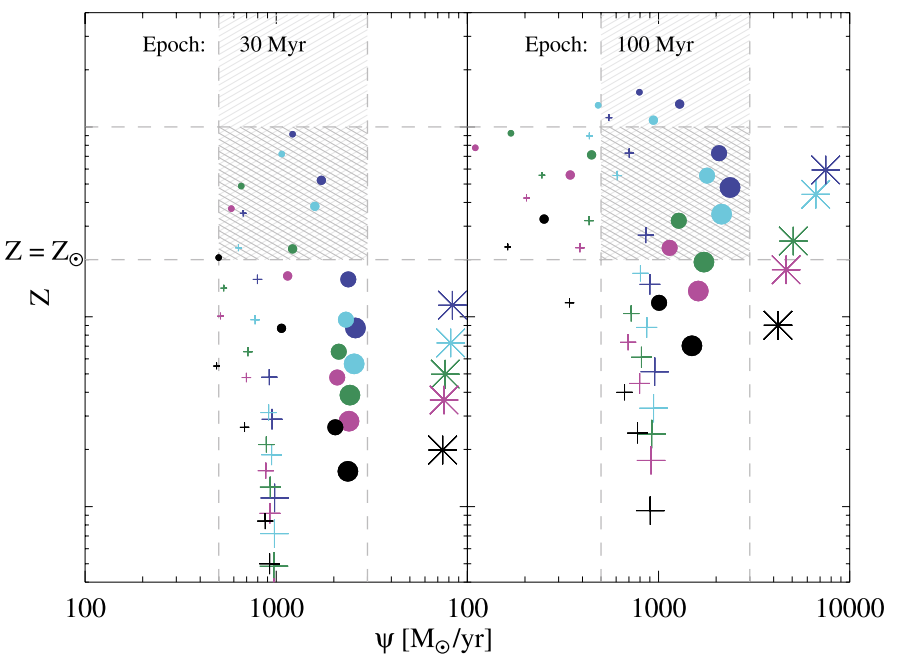

Fig. 3. Relation between metallicity and SFR at epochs of $30 \mathrm{Myr}$ (left panel) and $100 \mathrm{Myr}$ (right panel). The colored symbols are obtained for different initial gas masses, $M_{\text {ini }}$, SFRs, and IMFs. The size of the symbols is scaled by $M_{\text {ini }}$. Calculations are made for $M_{\text {ini }}=1.3 \times 10^{12} M_{\odot}$ (largest symbol), $M_{\text {ini }}=5 \times 10^{11} M_{\odot}, M_{\text {ini }}=3 \times 10^{11} M_{\odot}, M_{\text {ini }}=$ $1 \times 10^{11} M_{\odot}$, and $M_{\text {ini }}=5 \times 10^{10} M_{\odot}$ (smallest symbol). The crosses correspond to calculations for a initial SFR $\psi_{\text {ini }}=10^{3} M_{\odot} \mathrm{yr}^{-1}$, the filled circles to $\psi_{\text {ini }}=3 \times 10^{3} M_{\odot} \mathrm{yr}^{-1}$ and the stars to $\psi_{\text {ini }}=10^{4} M_{\odot} \mathrm{yr}^{-1}$. The black, green, cyan, magenta, and blue colors denote the Salpeter, massheavy, top-heavy, Larson 1, and Larson 2 IMF, respectively. The dark grey shaded region indicates the range of the metallicity and SFR based on observations of QSOs at $z>6$. The vertical dashed lines represent the lower and upper limits of the observationally derived SFRs. The light grey shaded area accounts for the uncertainty of the upper limit of the metallicity. The horizontal dashed lines mark the lower and possibly upper limit of the metallicity.

same $\psi_{\text {ini }}$ or with the lower initial SFR, and top-heavy IMFs are within the dark grey shaded region as well.

At an epoch of 100 Myr the metallicity has increased in all models, while the SFR in the less massive galaxies has significantly decreased. The models for $M_{\text {ini }}=3-5 \times 10^{11} M_{\odot}$, $\psi_{\text {ini }}=3 \times 10^{3} M_{\odot} \mathrm{yr}^{-1}$, and top heavy IMFs constitute the best results. In galaxies with $M_{\text {ini }}=3 \times 10^{11} M_{\odot}$, the same initial SFR, and either a mass-heavy or Larson $1 \mathrm{IMF}$ the obtained values for $Z$ and $\psi(t)$ are also in agreement with the observed values. The metallicities in the low mass galaxies which give the best agreement at $30 \mathrm{Myr}$ are now shifted above the upper limit, while the SFRs remain in the observed range. The models for a galaxy with $M_{\text {ini }}=1 \times 10^{11} M_{\odot}$, a lower initial SFR of $10^{3} M_{\odot} \mathrm{yr}^{-1}$, and top-heavy IMFs at this epoch (100 Myr) reach sufficiently high metallicities, while high enough SFRs are sustained.

\section{3. $\mathrm{CO}$ conversion factor and gas-to- $\mathrm{H}_{2}$ mass ratio}

To evaluate the calculated models, we additionally consider the relation between the gas-to- $\mathrm{H}_{2}$ mass ratio and the $\mathrm{CO}$ conversion factor used to derive the molecular gas mass in a galaxy.

Detections of high excitation CO line emission in QSOs at $z>$ (5)6 indicate the presence of $0.7-2.5 \times 10^{10} M_{\odot}$ of molecular hydrogen (e.g., Bertoldi et al. 2003b; Walter et al. 2003, 2004; Riechers et al. 2009; Wang et al. 2010). This molecular gas mass is derived from the relation $M_{\mathrm{H}_{2}}=\alpha \times$ $L_{\mathrm{CO}(1-0)}^{\prime}$, where $\alpha$ is the conversion factor between the low excitation $\mathrm{CO} J=1-0$ line luminosity $L_{\mathrm{CO}(1-0)}^{\prime}$ and $M_{\mathrm{H}_{2}}$. For spiral galaxies $\alpha$ is typically $\sim 4.6 M_{\odot}\left(\mathrm{K} \mathrm{km} \mathrm{s}^{-1} \mathrm{pc}^{2}\right)^{-1}$ (e.g.,
Solomon \& Barrett 1991), while for the centre of nearby ultra luminous starburst galaxies a conversion factor of $\alpha=0.8-1 M_{\odot}$ $\left(\mathrm{K} \mathrm{km} \mathrm{s}^{-1} \mathrm{pc}^{2}\right)^{-1}$ is appropriate (e.g., Downes \& Solomon 1998). The latter value of $\alpha$ is usually used for e.g., high- $z$ QSOs (e.g., Bertoldi et al. 2003b; Walter et al. 2003; Wang et al. 2010), Ultra Luminous Infrared Galaxies (ULIRGs) (Yan et al. 2010) or for high- $z$ sub-mm galaxies (SMGs) (Tecza et al. 2004; Greve et al. 2005). However $\alpha$ is not well known in the case of very high excitation.

In our models we have computed the total $(\mathrm{H}+\mathrm{He})$ gas mass $M_{\mathrm{G}}$ which remains in the galaxies at a given epoch. The molecular gas mass, $M_{\mathrm{H}_{2}}$, constitutes a certain fraction of the total gas mass, $M_{\mathrm{G}}$. Hence we introduce the gas-to- $\mathrm{H}_{2}$ mass ratio as $\eta_{\mathrm{g}, \mathrm{H}_{2}}=M_{\mathrm{G}} / M_{\mathrm{H}_{2}}$. The $\mathrm{CO}$ conversion factor can thereby be expressed as a function of $\eta_{\mathrm{g}, \mathrm{H}_{2}}$ as

$$
\alpha=\frac{M_{\mathrm{G}}}{\eta_{\mathrm{g}, \mathrm{H}_{2}} L_{\mathrm{CO}(1-0)}^{\prime}},
$$

where $\eta_{\mathrm{g}, \mathrm{H}_{2}} \geq 1$ is kept as a free parameter. In ULIRGs and SMGs a major fraction of the gas is believed to exist in form of molecular hydrogen (e.g., Sanders \& Mirabel 1996). For example a value for $\eta_{\mathrm{g}, \mathrm{H}_{2}}$ of $\sim 1$ has been found for the $z=3$ radio galaxy B3 J2330+3927 (De Breuck et al. 2003). This might also be the case for QSOs and suggests a gas-to- $\mathrm{H}_{2}$ ratio between 1 and 2 .

In Fig. 4 we show the results for $\alpha$ as a function of $\eta_{\mathrm{g}, \mathrm{H}_{2}}$ with $\psi_{\text {ini }}=3 \times 10^{3} M_{\odot} \mathrm{yr}^{-1}$ for models with $M_{\text {ini }} \leq 5 \times 10^{11} M_{\odot}$ and with $\psi_{\text {ini }}=10^{4} M_{\odot} \mathrm{yr}^{-1}$ for the most massive galaxy. Calculations are performed for two different epochs; $30 \mathrm{Myr}$ (top panel) and $100 \mathrm{Myr}$ (bottom panel). The IMFs involved are the top-heavy IMF and the Salpeter IMF. We adopt a CO line luminosity $L_{\mathrm{CO}(1-0)}^{\prime}=2.7 \times 10^{10} \mathrm{~K} \mathrm{~km} \mathrm{~s}^{-1} \mathrm{pc}^{2}$ which is based on the derived values of $\mathrm{J} 1148+5251$ and $\mathrm{J} 0840+5624$ (e.g., Bertoldi et al. 2003b; Walter et al. 2003; Wang et al. 2010).

The difference of $\alpha$ from calculations with a lower $L_{\mathrm{CO}(1-0)}^{\prime}$ (i.e., $L_{\mathrm{CO}(1-0)}^{\prime}=1.5 \times 10^{10} \mathrm{~K} \mathrm{~km} \mathrm{~s}^{-1} \mathrm{pc}^{2}$ ) is indicated by the arrow in Fig. 4. The grey shaded area signifies a possible range for $\alpha$ and $\eta_{\mathrm{g}, \mathrm{H}_{2}}$ as discussed above.

For a fixed value of $\alpha$ the gas-to- $\mathrm{H}_{2}$ ratio increases with increasing initial mass of the galaxy. This is as a consequence of the larger amounts of gas mass remaining in the more massive galaxies at the epochs of interest (see also Paper I). Conversely, for a fixed $\eta_{\mathrm{g}, \mathrm{H}_{2}}, \alpha$ increases with increasing $M_{\mathrm{ini}}$. The maximum value of $\alpha$ is obtained for $\eta_{\mathrm{g}, \mathrm{H}_{2}}=1$, i.e., $M_{\mathrm{G}} \equiv M_{\mathrm{H}_{2}}$. We find that at both epochs, the maximum value of $\alpha$ for the less massive galaxies is lower than $\sim 4.6 M_{\odot}\left(\mathrm{K} \mathrm{km} \mathrm{s}^{-1} \mathrm{pc}^{2}\right)^{-1}$. For a given $M_{\mathrm{ini}}, \alpha$, and $\eta_{\mathrm{g}, \mathrm{H}_{2}}$ are lower at later epochs. For a lower $L_{\mathrm{CO}(1-0)}^{\prime}, \alpha$ shifts to higher values for a given $\eta_{\mathrm{g}, \mathrm{H}_{2}}$.

At an epoch of $30 \mathrm{Myr}$ the values for $\alpha$ and $\eta_{\mathrm{g}, \mathrm{H}_{2}}$ are similar for all IMFs and galaxies with $M_{\text {ini }}>1 \times 10^{11} M_{\odot}$, while the difference becomes larger with decreasing $M_{\text {ini }}$. Feasible values of $\alpha$ and $\eta_{\mathrm{g}, \mathrm{H}_{2}}$ are possible for galaxies with $M_{\mathrm{ini}}=1 \times 10^{11} M_{\odot}$ and the higher value of $L_{\mathrm{CO}(1-0)}^{\prime}$. For top-heavy IMFs $\eta_{\mathrm{g}, \mathrm{H}_{2}}=1$ results in a maximum $\alpha$ of $\sim 2.3 M_{\odot}\left(\mathrm{K} \mathrm{km} \mathrm{s}^{-1} \mathrm{pc}^{2}\right)^{-1}$, while for $\alpha=0.8 M_{\odot}\left(\mathrm{K} \mathrm{km} \mathrm{s}^{-1} \mathrm{pc}^{2}\right)^{-1}$, the fraction of molecular hydrogen is about one third of the total gas mass. In the least massive galaxy $\left(M_{\text {ini }}=5 \times 10^{10} M_{\odot}\right)$ and for a top-heavy IMF $\alpha \approx 0.8 M_{\odot}$ $\left(\mathrm{K} \mathrm{km} \mathrm{s}^{-1} \mathrm{pc}^{2}\right)^{-1}$ presupposes that all the gas in this system is in the form of molecular hydrogen. In more massive systems with $M_{\text {ini }}=1-3 \times 10^{11} M_{\odot}$, a value of $\alpha \approx 0.8-1 M_{\odot}\left(\mathrm{K} \mathrm{km} \mathrm{s}^{-1} \mathrm{pc}^{2}\right)^{-1}$ presumes that the molecular hydrogen constitutes only a small fraction of about $1 / 10-1 / 20$ of the total gas mass. 


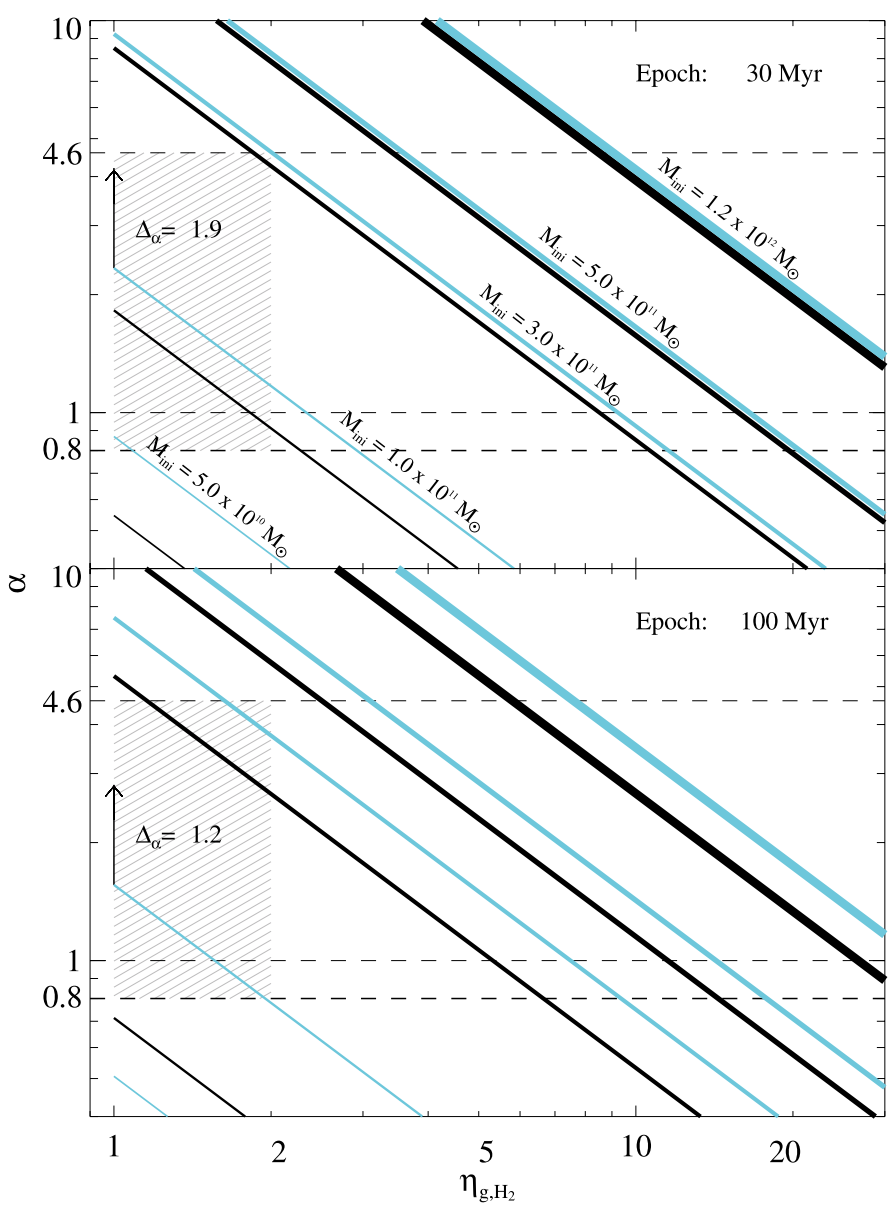

Fig. 4. $\mathrm{CO}$ conversion factor versus gas-to- $\mathrm{H}_{2}$ ratio at epochs $30 \mathrm{Myr}$ (top panel) and $100 \mathrm{Myr}$ (bottom panel). The solid lines correspond to calculations of $\alpha$ as a function of the gas-to- $\mathrm{H}_{2}$ ratio $\eta_{\mathrm{g}, \mathrm{H}_{2}}$ for a $\mathrm{CO}$ line luminosity of $L_{\mathrm{CO}(1-0)}^{\prime}=2.7 \times 10^{10} \mathrm{~K} \mathrm{~km} \mathrm{~s}^{-1} \mathrm{pc}^{2}$. Calculations are performed for different IMFs and galaxies for a range of different initial gas masses $M_{\text {ini }}$. The thickness of the lines is scaled by $M_{\text {ini }}$ as indicated in the upper panel. The black and cyan colors denote the Salpeter and top-heavy IMF, respectively. The arrow indicates the shift of $\alpha$ for calculations with the lower $L_{\mathrm{CO}(1-0)}^{\prime}=1.5 \times 10^{10} \mathrm{~K} \mathrm{~km} \mathrm{~s}^{-1} \mathrm{pc}^{2}$, and $\Delta_{\alpha}$ is the difference of $\alpha$ between the higher and lower $L_{\mathrm{CO}(1-0)}^{\prime}$. Calculations are shown for models with $\psi_{\text {ini }}=3 \times 10^{3} M_{\odot} \mathrm{yr}^{-1}$, except for the model for the most massive galaxy for which $\psi_{\text {ini }}=10^{4} M_{\odot} \mathrm{yr}^{-1}$. The grey shaded region signifies the possible range of $\alpha$ and $\eta_{\mathrm{g}, \mathrm{H}_{2}}$. The horizontal black dashed lines mark the values of $\alpha=0.8,1$ and $4.6 M_{\odot}\left(\mathrm{K} \mathrm{km} \mathrm{s}^{-1} \mathrm{pc}^{2}\right)^{-1}$.

At an epoch of 100 Myr a clear separation between the IMFs is noticeable. For a Salpeter IMF the galaxies underwent a stronger gas exhaustion than for a top-heavy IMF, which is more significant for the less massive galaxies. As for the epoch at $30 \mathrm{Myr}$ the system with $M_{\text {ini }}=1 \times 10^{11} M_{\odot}$ and top-heavy IMF is plausible, i.e., for $\alpha \sim 0.8 M_{\odot}\left(\mathrm{K} \mathrm{km} \mathrm{s}^{-1} \mathrm{pc}^{2}\right)^{-1}$ the gas-to- $\mathrm{H}_{2}$ ratio $\eta_{\mathrm{g}, \mathrm{H}_{2}}=2$. For the galaxies with $M_{\text {ini }}=3-5 \times 10^{11} M_{\odot}$ and top-heavy IMF we obtain $\alpha=1.4-1.5$ for a corresponding gasto- $\mathrm{H}_{2}$ ratio $\eta_{\mathrm{g}, \mathrm{H}_{2}}=5-10$, resulting in a molecular mass of $M_{\mathrm{H}_{2}}$ $\sim 3.7 \times 10^{10} M_{\odot}$. Alternatively, a higher value for $\alpha$ up to $4.6 \mathrm{re}-$ sults in a lower $\eta_{\mathrm{g}, \mathrm{H}_{2}}=2-4$. It is noteworthy that for the assumed $L_{\mathrm{CO}(1-0)}^{\prime}=2.7 \times 10^{10} \mathrm{~K} \mathrm{~km} \mathrm{~s}^{-1} \mathrm{pc}^{2}, \alpha=4.6 M_{\odot}\left(\mathrm{K} \mathrm{km} \mathrm{s}^{-1} \mathrm{pc}^{2}\right)^{-1}$ implies $M_{\mathrm{H}_{2}}=1.2 \times 10^{11} M_{\odot}$. The likelihood that such a high $M_{\mathrm{H}_{2}}$ could have been built up within a short timescale of 30$100 \mathrm{Myr}$ however is unclear.

\section{Discussion}

\subsection{Individual QSOs at $z \gtrsim 6$}

We ascertain plausible scenarios by comparing the model results discussed in Sect. 3 with the derived values from observations for specific quantities of individual QSOs listed in Table 2. The calculated values for diverse properties such as $M_{\mathrm{d}}, M_{*}, M_{\mathrm{H}_{2}}$, metallicity, and SFR from the models discussed below, which best match the QSOs, are listed in Table 3. The corresponding model parameters, and all models which match the discussed properties within the range defined by observations, are summarized in Table 4.

We find that at an epoch of $30 \mathrm{Myr}$ the models with an initial mass of the galaxy of $M_{\text {ini }}=1 \times 10^{11} M_{\odot}$, an initial SFR of $\psi_{\text {ini }}=3 \times 10^{3} M_{\odot} \mathrm{yr}^{-1}$ and either a Larson $2 \mathrm{IMF}$, a top-heavy or a mass-heavy IMF reproduce the observed quantities of some QSOs at $z>6$ in the case of a "maximum" SN efficiency.

In particular, the model with a top-heavy IMF is best applicable to the QSO J1148+5251. The amount of dust reached is between $3.1-5.1 \times 10^{8} M_{\odot}$ for dust destruction in the ISM with $M_{\mathrm{cl}}=100-0 M_{\odot}$. A stellar mass of $M_{*} \sim 3.5 \times 10^{10} M_{\odot}$ is obtained. The metallicity in the system is $\sim 2 Z_{\odot}$ and a SFR of $\sim 1600 M_{\odot} \mathrm{yr}^{-1}$ could be sustained. This model is also favored given its values of $\alpha$ and $\eta_{\mathrm{g}, \mathrm{H}_{2}}$. The higher $\mathrm{H}_{2}$ mass of $M_{\mathrm{H}_{2}}=3.7 \times 10^{10} M_{\odot}$ derived by Riechers et al. (2009) leads to $\eta_{\mathrm{g}, \mathrm{H}_{2}}<2$ and $\alpha \sim 1.4 M_{\odot}\left(\mathrm{K} \mathrm{km} \mathrm{s}^{-1} \mathrm{pc}^{2}\right)^{-1}$. However, such a galaxy with $M_{\text {ini }}=1 \times 10^{11} M_{\odot}$ implies that the dynamical mass is larger than the derived $M_{\text {dyn }}$ of $\sim 5.5 \times 10^{10} M_{\odot}$ (for a $i=65^{\circ}$ ) by Walter et al. (2004). While none of the models for $M_{\text {ini }}=5 \times 10^{10} M_{\odot}$, which was used by Dwek et al. (2007), can be applied, a lower inclination angle similar to what has been adopted for the other QSOs might be considered.

Another possible match with the properties of $\mathrm{J} 1148+5251$ is achieved by the same set of values for $M_{\text {ini }}, \psi_{\text {ini }}$, SN efficiency and IMF at an epoch of 100 Myr. The calculated stellar mass is within the estimated range from observations and the dust mass is $\sim 2.4-8.9 \times 10^{8} M_{\odot}$, depending on $M_{\mathrm{cl}}$. However, the SFR dropped to $\sim 1000 M_{\odot} \mathrm{yr}^{-1}$, while the metallicity increased to $\sim 5 Z_{\odot}$. In view of the lower SFR reached by these models than suggested by observations at epochs either 30 or $100 \mathrm{Myr}$, a higher initial SFR than the $3 \times 10^{3} M_{\odot} \mathrm{yr}^{-1}$ might be conceivable. In Fig. 3 one notices that a longer evolution with the same (or lower) initial SFR as used here does not lead to a better agreement with observations, since this results in an even lower SFR and higher metallicity.

In view of this we find that this scenario at an epoch of $100 \mathrm{Myr}$ is more appropriate for the QSOs J1048+4637 (Fan et al. 2003) at $z=6.23$ and J2054-0005 (Jiang et al. 2008) at $z=6.06$. For the latter QSO a fine tuning of the epoch to 70 Myr results in a better match. At this epoch we obtain a SFR of $1150 M_{\odot} \mathrm{yr}^{-1}$ and a metallicity of $\sim 4.4 Z_{\odot}$. The amount of dust is $M_{\mathrm{d}} \sim 2.7 \times 10^{8} M_{\odot}$ (for $M_{\mathrm{cl}}=100 M_{\odot}$ ), while the stellar mass is $M_{*} \sim 4.7 \times 10^{10} M_{\odot}$. The lower derived $L_{\mathrm{CO}(1-0)}^{\prime}$ leads to $\eta_{\mathrm{g}, \mathrm{H}_{2}} \sim 3-4$ in case $\alpha=0.8-1 M_{\odot}\left(\mathrm{K} \mathrm{km} \mathrm{s}^{-1} \mathrm{pc}^{2}\right)^{-1}$ is applied, while for $\eta_{\mathrm{g}, \mathrm{H}_{2}} \sim 2$ a value for $\alpha$ of $\sim 1.6$ would be required. For $\mathrm{J} 1048+4637$ the model for a lower initial SFR of $\psi_{\text {ini }}=10^{3} M_{\odot} \mathrm{yr}^{-1}$ might be an option. The SFR is $\sim 610 M_{\odot} \mathrm{yr}^{-1}$ and the metallicity is $\sim 3.4 Z_{\odot}$. While the stellar mass remains low, $M_{*} \sim 2.8 \times 10^{10} M_{\odot}$, a dust mass of $M_{\mathrm{d}} \sim 3.5$ $\times 10^{8} M_{\odot}$ is obtained for a "maximum" SN efficiency and moderate dust destruction in the ISM. However, for $\alpha=0.8-1 M_{\odot}$ $\left(\mathrm{K} \mathrm{km} \mathrm{s}^{-1} \mathrm{pc}^{2}\right)^{-1}$ the gas-to- $\mathrm{H}_{2}$ ratio is $\sim 5-6$, since for the lower initial SFR the system at this epoch is less exhausted. 
C. Gall et al.: Genesis and evolution of dust in galaxies in the early Universe. II.

Table 2. Observed properties of quasars at $z \gtrsim 6.0$.

\begin{tabular}{lccccccc}
\hline \hline Object & $z$ & $\begin{array}{c}L_{\mathrm{CO}(1-0)}^{\prime} \\
10^{10} \mathrm{~K} \mathrm{~km} \mathrm{~s}^{-1} \mathrm{pc}^{2}\end{array}$ & $\begin{array}{c}S F R \\
M_{\odot} \mathrm{yr}^{-1}\end{array}$ & $\begin{array}{c}M_{\mathrm{d}} \\
10^{8} M_{\odot}\end{array}$ & $\begin{array}{c}M_{\mathrm{H}_{2}} \\
10^{10} M_{\odot}\end{array}$ & $\begin{array}{c}M_{\mathrm{dyn}} \sin ^{2} i \\
10^{10} M_{\odot}\end{array}$ & Ref. \\
\hline $\mathrm{J} 1148+5251$ & 6.42 & $3.0 \pm 0.3$ & 2380 & $5.9 \pm 0.7$ & $2.4 / 3.7^{a}$ & 4.5 & $1,2,3,4$ \\
$\mathrm{~J} 1048+4637$ & 6.23 & $1.2 \pm 0.2$ & 650 & $4.3 \pm 0.6$ & 1.0 & 4.5 & $1,2,3$ \\
$\mathrm{~J} 2054-0005$ & 6.06 & $1.5 \pm 0.3$ & 1180 & $3.4 \pm 0.8$ & 1.2 & 4.2 & $5,2,3$ \\
$\mathrm{~J} 0840+5624$ & 5.85 & $3.2 \pm 0.4$ & 1460 & $4.7 \pm 0.9$ & 2.5 & 24.2 & $6,2,3$ \\
\hline
\end{tabular}

References. (1) Fan et al. (2003); (2) Wang et al. (2010); (3) Michałowski et al. (2010); (4) Walter et al. (2004); (5) Jiang et al. (2008); (6) Fan et al. (2006).

Notes. ${ }^{(a)} M_{\mathrm{H}_{2}}=3.7 \times 10^{10} M_{\odot}$ deduced from [C I line detections by Riechers et al. (2009).

Table 3. Calculated properties from the best matching models of $z \gtrsim 6$ QSOs from our sample.

\begin{tabular}{|c|c|c|c|c|c|c|c|}
\hline Object $^{a}$ & $\begin{array}{c}S F R \\
M_{\odot} \mathrm{yr}^{-1}\end{array}$ & $\begin{array}{c}M_{\mathrm{d}} \\
10^{8} M_{\odot}\end{array}$ & $\begin{array}{c}M_{*} \\
10^{10} M_{\odot}\end{array}$ & $\begin{array}{c}Z \\
Z_{\odot}\end{array}$ & $\alpha^{b}$ & $\eta_{\mathrm{g}, \mathrm{H}_{2}}{ }^{b}$ & $\begin{array}{l}M_{\mathrm{H}_{2}}{ }^{b} \\
10^{10} M_{\odot}\end{array}$ \\
\hline $\mathrm{J} 1148+5251(\mathrm{~A})$ & 1600 & $3.1-5.1$ & 3.5 & 2 & $0.8-2.3$ & $3.0-1.0$ & $2.16-6.2$ \\
\hline $\mathrm{J} 1148+5251(\mathrm{~B})$ & 1000 & $2.4-8.9$ & 5.4 & 5 & $0.8-1.55$ & $2.0-1.0$ & $2.10-4.1$ \\
\hline $\mathrm{J} 1048+4637(\mathrm{~A})$ & 1000 & $2.4-8.9$ & 5.4 & 5 & $0.8-2.8$ & $3.4-1.0$ & $1.2-4.2$ \\
\hline $\mathrm{J} 1048+4637(\mathrm{~B})$ & 610 & 3.5 & 2.8 & 3.4 & $0.8-4.5$ & $5.8-1.0$ & $1.2-6.7$ \\
\hline $\mathrm{J} 2054-0005$ & 1150 & 2.7 & 4.7 & 4.4 & $0.8-3.2$ & $3.0-1.0$ & $1.2-4.8$ \\
\hline $\mathrm{J} 0840+5624(\mathrm{~A})$ & 1500 & 2.1 & 11.0 & 4 & $0.8-4.6$ & $7.0-1.2$ & $2.5-14.7$ \\
\hline J0840+5624(B) & 1400 & 4.8 & 20.0 & 5 & $0.8-4.6$ & $10-1.8$ & $2.5-14.7$ \\
\hline
\end{tabular}

Notes. ${ }^{(a)}$ All models are calculated for a top-heavy IMF. Capital letters in brackets (A, B) signify the different models (see corresponding models in Table 4) for the same object. ${ }^{(b)}$ The ranges of $\eta_{\mathrm{g}, \mathrm{H}_{2}}$ and $M_{\mathrm{H}_{2}}$ corresponds to the range of $\alpha$, which is between the commonly used value of $\alpha=0.8$ and the possible upper limit.

At either the same or a later epoch the more massive galaxies with $M_{\text {ini }}=3-5 \times 10^{11} M_{\odot}$, an initial SFR of $\psi_{\text {ini }}=$ $3 \times 10^{3} M_{\odot} \mathrm{yr}^{-1}$ and IMFs biased towards higher stellar masses are applicable to some $z \sim 6$ QSOs. The stellar mass, metallicity, and SFR of these systems are in agreement with observations, with either top-heavy IMFs or a mass-heavy IMF leading to the best results. The amount of dust can be produced by SNe with a "high" SN efficiency and $M_{\mathrm{cl}} \leq 100 M_{\odot}$, although the dust masses reached are at the lower limit.

At an epoch of $170 \mathrm{Myr}$ the system with $M_{\mathrm{ini}}=3 \times 10^{11} M_{\odot}$ is plausible for the QSO J0840+5624 (Fan et al. 2006) at $z=5.85$, if an inclination angle higher than the assumed $40^{\circ}$ is assumed. The SFR is $\sim 1500 M_{\odot} \mathrm{yr}^{-1}$ and the metallicity is $\sim 4 Z_{\odot}$. The stellar mass is around $1.1 \times 10^{11} M_{\odot}$. The amount of dust obtained with a "high" SN efficiency is $2.1 \times 10^{8} M_{\odot}$, while with the "maximum" SN efficiency the dust mass exceeds a few times $10^{9} M_{\odot}$ (as already at an epoch of $100 \mathrm{Myr}$ ). However, for a $L_{\mathrm{CO}(1-0)}^{\prime}=3.2 \times 10^{10} \mathrm{~K} \mathrm{~km} \mathrm{~s}^{-1} \mathrm{pc}^{2}$ as derived for this QSO the gas-to- $\mathrm{H}_{2}$ ratio of $\eta_{\mathrm{g}, \mathrm{H}_{2}} \sim 5-7$ for $\alpha=0.8-1 M_{\odot}\left(\mathrm{K} \mathrm{km} \mathrm{s}^{-1} \mathrm{pc}^{2}\right)^{-1}$ is higher than for the less massive galaxies. In case of a lower $\eta_{\mathrm{g}, \mathrm{H}_{2}}$ of $\sim 2, \alpha \sim 2.7 M_{\odot}\left(\mathrm{K} \mathrm{km} \mathrm{s}^{-1} \mathrm{pc}^{2}\right)^{-1}$ is required. The larger galaxy with $M_{\text {ini }}=5 \times 10^{11} M_{\odot}, \psi_{\text {ini }}=3 \times 10^{3} M_{\odot} \mathrm{yr}^{-1}$ and top heavy IMF can account for the observed quantities at an epoch of 400 Myr. The amount of dust reached with a "high" SN efficiency is $\sim 4.8 \times 10^{8} M_{\odot}$ and the SFR is $\sim 1400 M_{\odot} \mathrm{yr}^{-1}$. The metallicity and stellar mass are in agreement, but the fraction of $M_{\mathrm{H}_{2}}$ is around $1 / 10$ for $\alpha=0.8 M_{\odot}\left(\mathrm{K} \mathrm{km} \mathrm{s}^{-1} \mathrm{pc}^{2}\right)^{-1}$, while $\alpha \sim 4 M_{\odot}\left(\mathrm{K} \mathrm{km} \mathrm{s}^{-1} \mathrm{pc}^{2}\right)^{-1}$ is needed for $\eta_{\mathrm{g}, \mathrm{H}_{2}}$ of $\sim 2$. A higher amount of $M_{\mathrm{H}_{2}}$ as denoted by the higher value of $\alpha$ in these massive galaxies might be possible. For example, the presence of large amounts of cold and low-excited molecular gas have been suggested by Papadopoulos et al. (2001) for the QSO APM $08279+5255$ at $z=3.91$.

\section{2. $S N$ efficiency and mass of the galaxy}

Our calculations show that with increasing $M_{\text {ini }}$ (and fixed $\psi_{\text {ini }}$, IMF) the SN dust production efficiencies can either be lowered or the degree of dust destruction increased in order to reach the required large dust masses. This is best demonstrated by models for the most massive galaxies with $M_{\mathrm{ini}}=3-13 \times 10^{11} M_{\odot}$ in which a "high" SN efficiency is sufficient in case of moderate to no dust destruction.

However, the largest system with $M_{\text {ini }}=1.3 \times 10^{12} M_{\odot}$ exceeds the plausible dynamical masses derived from observations of QSOs at $z \gtrsim(5) 6$ by more than an order of magnitude. Moreover, our computed models show that at least one of the properties of either SFR, $Z$ or $M_{*}$ are not in agreement with observations at any epoch for any assumption of either the initial SFR or the IMF (see also Paper I). Additionally the values for $\eta_{\mathrm{g}, \mathrm{H}_{2}}$ remain very high even for $\alpha=4.6 M_{\odot}\left(\mathrm{K} \mathrm{km} \mathrm{s}^{-1} \mathrm{pc}^{2}\right)^{-1}$. We therefore conclude that such a massive system as advocated by Valiante et al. (2009), cannot be applied to QSOs at $z>(5) 6$. Although systems with $M_{\text {ini }}=3-5 \times 10^{11} M_{\odot}$ are appropriate for some QSOs at $z<6$, such massive systems can only be applied to QSOs $>6$ when the inclination angle is lower than the assumed average angle.

The models which best reproduce the observed properties of QSOs $>6$ are for a galaxy with $M_{\mathrm{ini}}=1 \times 10^{11} M_{\odot}$, but necessitate a "maximum" SN efficiency and/or a moderate amount of dust destruction. The overall rapid evolution of dust and some properties in these models indicates that such QSOs could possibly be present at a higher redshift than $z>6.4$. An interesting example at a lower redshift of $z=1.135$ is the ULIRG SST J1604+4304, which shows properties similar to the considered high- $z$ QSOs. Kawara et al. (2010) reported a dust mass in this ULIRG of $1-2 \times 10^{8} M_{\odot}$, a metallicity of around $2.5 Z_{\odot}$ and estimated the age of the stellar population to be 40-200 Myr. 
Table 4. Models ${ }^{a}$ which match the observed range of properties of $z \geq 5$ QSOs.

\begin{tabular}{|c|c|c|c|c|c|c|}
\hline $\begin{array}{l}\text { Epoch } \\
M_{\odot}\end{array}$ & $\begin{array}{l}M_{\text {ini }} \\
10^{3} M_{\odot} \mathrm{yr}^{-1}\end{array}$ & $\psi_{\text {ini }}$ & $\begin{array}{c}\text { SN efficiency } \\
M_{\odot}\end{array}$ & $M_{\mathrm{cl}}$ & IMF & $z \gtrsim 6$ QSOs from our sample ${ }^{b}$ \\
\hline \multirow[t]{8}{*}{$30 \mathrm{Myr}$} & $5 \times 10^{10}$ & 3 & $\max$ & 100 & top-heavy, Larson 1, 2, mass-heavy & \\
\hline & & 3 & $\max$ & 0 & top-heavy, Larson 1, 2 & \\
\hline & & 3 & $\max$ & $\mathbf{0}$ & mass-heavy & \\
\hline & & 1 & $\max$ & 0,100 & top-heavy, Larson 2 & \\
\hline & $1 \times 10^{11}$ & 3 & $\max$ & 100 & Larson 2, mass-heavy & \\
\hline & & 3 & $\max$ & 0 & mass-heavy & \\
\hline & & 3 & $\max$ & $100-0$ & top-heavy & $\mathrm{J} 1148+5251(\mathrm{~A})$ \\
\hline & & 3 & high & 0 & Larson 2 & \\
\hline $70 \mathrm{Myr}$ & $1 \times 10^{11}$ & 1 & $\max$ & 100-0 & top-heavy & J2054-0005 \\
\hline \multirow[t]{21}{*}{$100 \mathrm{Myr}$} & $5 \times 10^{10}$ & 3 & $\max$ & 100 & Larson 2 & \\
\hline & & 3 & $\max$ & $\mathbf{0}$ & top-heavy, Larson 2 & \\
\hline & & 1 & $\max$ & 100 & Larson 2 & \\
\hline & $1 \times 10^{11}$ & 3 & $\max$ & 100 & Larson 2 & \\
\hline & & 3 & high & 0 & Larson 2 & \\
\hline & & 3 & $\max$ & 100-0 & top-heavy & $\mathrm{J} 1148+5251(\mathrm{~B}), \mathrm{J} 1048+4637(\mathrm{~A})$ \\
\hline & & 1 & $\max$ & 100 & Larson 2 & \\
\hline & & 1 & $\max$ & $100-0$ & top-heavy & $\mathrm{J} 1048+4637(\mathrm{~B})$ \\
\hline & $3 \times 10^{11}$ & 3 & $\max$ & 800 & Larson 1, mass-heavy & \\
\hline & & 3 & $\max$ & 800 & top-heavy, Larson 2 & \\
\hline & & 3 & $\max$ & 100 & Larson 1, mass-heavy & \\
\hline & & 3 & high & 0 & mass-heavy & \\
\hline & & 3 & high & $\mathbf{0}$ & top-heavy, Larson 2 & \\
\hline & & 1 & $\max$ & 800 & Larson 2 & \\
\hline & & 1 & high & 0 & Larson 2 & \\
\hline & $5 \times 10^{11}$ & 3 & $\max$ & 800 & mass-heavy & \\
\hline & & 3 & $\max$ & 800 & top-heavy, Larson 2 & \\
\hline & & 3 & high & 100 & top-heavy, mass-heavy & \\
\hline & & 3 & high & 100 & Larson 2 & \\
\hline & & 3 & high & 0 & mass-heavy & \\
\hline & & 3 & high & $\mathbf{0}$ & top-heavy, Larson 2 & \\
\hline $170 \mathrm{Myr}$ & $3 \times 10^{11}$ & 1 & high & 100-0 & top-heavy & $\mathrm{J} 0840+5624(\mathrm{~A})$ \\
\hline $400 \mathrm{Myr}$ & $5 \times 10^{11}$ & 1 & high & $100-0$ & top-heavy & $\mathrm{J} 0840+5624(\mathrm{~B})$ \\
\hline
\end{tabular}

Notes. ${ }^{(a)}$ All models which match the observed range of all properties of $z \geq 6$ QSOs are marked in bold face. ${ }^{(b)}$ Capital letters in brackets signify that different models $(\mathrm{A}, \mathrm{B})$ are applicable for the same object

The possibility of moderate dust destruction in the ISM was already discussed in Paper I. We found that the amount of dust for most models better coincide with observations for $M_{\mathrm{cl}} \leq 100 M_{\odot}$, which would be in agreement with the values of $M_{\mathrm{cl}}$ of $50-70 M_{\odot}$ derived for a multiphase ISM (e.g., McKee 1989; Dwek et al. 2007).

The "maximum" SN efficiency might be problematic. There is only little observational evidence that $\mathrm{SNe}$ can be very efficient (e.g., Wilson \& Batrla 2005; Douvion et al. 2001; Dunne et al. 2009), and theoretical models predict significant dust destruction in reverse shocks of SNe (e.g., Bianchi \& Schneider 2007; Nozawa et al. 2007, 2010). On the other hand, these models also show that the effectiveness of dust destruction depends on various properties such as the geometry of the shocks, the density of the ejecta and the ISM, the size and shape of the grains, clumping in the SNe ejecta, and different SN types. In addition there is some observational evidence that type IIn SNe and sources such as luminous blue variables are possibly efficient dust producers (Fox et al. 2009; Smith et al. 2009; Gomez et al. 2010). While dust production and destruction in SNe is yet unresolved, a "maximum" SN efficiency cannot be ruled out (e.g., Gall et al. in prep). Alternatively, either dust formation in the outflowing winds of QSOs or grain growth in the ISM might be an option (e.g., Elvis et al. 2002; Dwek et al. 2007; Draine 2009; Michałowski et al. 2010; Pipino et al. 2011; Dwek \& Cherchneff 2011) as supplementary or primary dust sources. However it remains to be investigated, if dust grain growth can be as efficient as required under the prevailing conditions of high star formation activity and a short time span. Typical grain growth timescales in molecular clouds are of order $10^{7} \mathrm{yr}$, but depending on the density and metallicity these can possibly be shorter (e.g., Hirashita 2000; Zhukovska et al. 2008; Draine 2009). The fact that the starburst is assumed to occur in an initially dust free galaxy implies that heavy elements first need to be ejected into the ISM before grain growth can take place. In forthcoming work we will further develop the model to investigate the impact of different infall and outflow scenarios on the evolution of the amount of dust and various properties of a galaxy.

Acknowledgements. We would like to thank Michal Michałowski, Darach Watson, Thomas Greve, and Sabine König for informative and helpful discussions. We also thank the anonymous referee for useful suggestions which helped improve the paper. The Dark Cosmology Centre is funded by the DNRF.

\section{References}

Barth, A. J., Martini, P., Nelson, C. H., \& Ho, L. C. 2003, ApJ, 594, L95 Becker, G. D., Sargent, W. L. W., Rauch, M., \& Simcoe, R. A. 2006, ApJ, 640, 69

Beelen, A., Cox, P., Benford, D. J., et al. 2006, ApJ, 642, 694

Bertoldi, F., \& Cox, P. 2002, A\&A, 384, L11

Bertoldi, F., Carilli, C. L., Cox, P., et al. 2003a, A\&A, 406, L55

Bertoldi, F., Cox, P., Neri, R., et al. 2003b, A\&A, 409, L47

Bianchi, S., \& Schneider, R. 2007, MNRAS, 378, 973 
C. Gall et al.: Genesis and evolution of dust in galaxies in the early Universe. II.

Carilli, C. L., Bertoldi, F., Rupen, M. P., et al. 2001, ApJ, 555, 625 Carilli, C. L., Walter, F., Bertoldi, F., et al. 2004, AJ, 128, 997 De Breuck, C., Neri, R., Morganti, R., et al. 2003, A\&A, 401, 911

Di Matteo, T., Croft, R. A. C., Springel, V., \& Hernquist, L. 2004, ApJ, 610, 80

Dietrich, M., Hamann, F., Appenzeller, I., \& Vestergaard, M. 2003, ApJ, 596, 817

Douvion, T., Lagage, P. O., Cesarsky, C. J., \& Dwek, E. 2001, A\&A, 373, 281

Downes, D., \& Solomon, P. M. 1998, ApJ, 507, 615

Draine, B. T. 2009, in ASP Conf. Ser. 414, ed. T. Henning, E. Grün, \& J. Steinacker, 453

Dunne, L., Maddox, S. J., Ivison, R. J., et al. 2009, MNRAS, 394, 1307

Dwek, E. 1998, ApJ, 501, 643

Dwek, E., \& Cherchneff, I. 2011, ApJ, 727, 63

Dwek, E., Galliano, F., \& Jones, A. P. 2007, ApJ, 662, 927

Eldridge, J. J., Izzard, R. G., \& Tout, C. A. 2008, MNRAS, 384, 1109

Elvis, M., Marengo, M., \& Karovska, M. 2002, ApJ, 567, L107

Fan, X., Strauss, M. A., Schneider, D. P., et al. 2003, AJ, 125, 1649

Fan, X., Strauss, M., Richards, G., et al. 2004, in AGN Physics with the Sloan Digital Sky Survey, ed. G. T. Richards \& P. B. Hall, ASP Conf. Ser., 311, 431

Fan, X., Strauss, M. A., Becker, R. H., et al. 2006, AJ, 132, 117

Fox, O., Skrutskie, M. F., Chevalier, R. A., et al. 2009, ApJ, 691, 650

Freudling, W., Corbin, M. R., \& Korista, K. T. 2003, ApJ, 587, L67

Gall, C., Andersen, A. C., \& Hjorth, J. 2011, A\&A, 528, A13 (Paper I)

Gallerani, S., Maiolino, R., Juarez, Y., et al. 2010, A\&A, 523, A85

Georgy, C., Meynet, G., Walder, R., Folini, D., \& Maeder, A. 2009, A\&A, 502, 611

Gomez, H. L., Dunne, L., Ivison, R. J., et al. 2009, MNRAS, 397, 1621

Gomez, H. L., Vlahakis, C., Stretch, C. M., et al. 2010, MNRAS, 401, L48

Greve, T. R., Bertoldi, F., Smail, I., et al. 2005, MNRAS, 359, 1165

Guedes, J., Madau, P., Mayer, L., \& Callegari, S. 2010, ApJ, accepted [arXiv: 1008.2032]

Hao, H., Elvis, M., Civano, F., et al. 2010a, ApJ, 724, L59

Hao, H., Elvis, M., Civano, F., \& Lawrence, A. 2010b [arXiv: 1011. 0429]

Hines, D. C., Krause, O., Rieke, G. H., et al. 2006, ApJ, 641, L85

Hirashita, H. 2000, PASJ, 52, 585

Hirashita, H., Nozawa, T., Kozasa, T., Ishii, T. T., \& Takeuchi, T. T. 2005, MNRAS, 357, 1077

Jiang, L., Fan, X., Hines, D. C., et al. 2006, AJ, 132, 2127

Jiang, L., Fan, X., Annis, J., et al. 2008, AJ, 135, 1057

Jiang, L., Fan, X., Brandt, W. N., et al. 2010, Nature, 464, 380

Juarez, Y., Maiolino, R., Mujica, R., et al. 2009, A\&A, 494, L25

Kawakatu, N., \& Wada, K. 2009, ApJ, 706, 676

Kawara, K., Oyabu, S., Matsuoka, Y., et al. 2010, MNRAS, 402, 335

Kennicutt, Jr., R. C. 1998, ApJ, 498, 541

Larson, R. B. 1998, MNRAS, 301, 569

Leipski, C., Meisenheimer, K., Klaas, U., et al. 2010, A\&A, 518, L34

Li, Y., Hernquist, L., Robertson, B., et al. 2007, ApJ, 665, 187
Maiolino, R., Juarez, Y., Mujica, R., Nagar, N. M., \& Oliva, E. 2003, ApJ, 596, L155

Maiolino, R., Schneider, R., Oliva, E., et al. 2004, Nature, 431, 533

Maiolino, R., Cox, P., Caselli, P., et al. 2005, A\&A, 440, L51

Maiolino, R., Nagao, T., Marconi, A., et al. 2006, Mem. Soc. Astron. Ital., 77, 643

Marconi, A., \& Hunt, L. K. 2003, ApJ, 589, L21

McKee, C. 1989, in Interstellar Dust, ed. L. J. Allamandola, \& A. G. G. M. Tielens, IAU Symp., 135, 431

Michałowski, M. J., Murphy, E. J., Hjorth, J., et al. 2010, A\&A, 522, A15

Morgan, H. L., \& Edmunds, M. G. 2003, MNRAS, 343, 427

Nomoto, K., Tominaga, N., Umeda, H., Kobayashi, C., \& Maeda, K. 2006, Nuclear Phys. A, 777, 424

Nozawa, T., Kozasa, T., Habe, A., et al. 2007, ApJ, 666, 955

Nozawa, T., Kozasa, T., Tominaga, N., et al. 2010, ApJ, 713, 356

Omont, A., Cox, P., Bertoldi, F., et al. 2001, A\&A, 374, 371

Omont, A., Beelen, A., Bertoldi, F., et al. 2003, A\&A, 398, 857

Papadopoulos, P., Ivison, R., Carilli, C., \& Lewis, G. 2001, Nature, 409, 58

Pipino, A., Fan, X. L., Matteucci, F., et al. 2011, A\&A, 525, A61

Polletta, M., Courvoisier, T., Hooper, E. J., \& Wilkes, B. J. 2000, A\&A, 362, 75

Riechers, D. A., Walter, F., Carilli, C. L., \& Bertoldi, F. 2007, ApJ, 671, L13

Riechers, D. A., Walter, F., Bertoldi, F., et al. 2009, ApJ, 703, 1338

Robson, I., Priddey, R. S., Isaak, K. G., \& McMahon, R. G. 2004, MNRAS, 351 , L29

Salpeter, E. E. 1955, ApJ, 121, 161

Sanders, D. B., \& Mirabel, I. F. 1996, ARA\&A, 34, 749

Smith, N., Silverman, J. M., Chornock, R., et al. 2009, ApJ, 695, 1334

Solomon, P. M. \& Barrett, J. W. 1991, in Dynamics of Galaxies and Their Molecular Cloud Distributions, ed. F. Combes, \& F. Casoli, IAU Symp., 146, 235

Tecza, M., Baker, A. J., Davies, R. I., et al. 2004, ApJ, 605, L109

Valiante, R., Schneider, R., Bianchi, S., \& Andersen, A. C. 2009, MNRAS, 397, 1661

van den Hoek, L. B., \& Groenewegen, M. A. T. 1997, A\&AS, 123, 305

Vestergaard, M. 2004, ApJ, 601, 676

Walter, F., Bertoldi, F., Carilli, C., et al. 2003, Nature, 424, 406

Walter, F., Carilli, C., Bertoldi, F., et al. 2004, ApJ, 615, L17

Walter, F., Riechers, D., Cox, P., et al. 2009, Nature, 457, 699

Wang, R., Carilli, C. L., Wagg, J., et al. 2008, ApJ, 687, 848

Wang, R., Carilli, C. L., Neri, R., et al. 2010, ApJ, 714, 699

Willott, C. J., McLure, R. J., \& Jarvis, M. J. 2003, ApJ, 587, L15

Willott, C. J., Delorme, P., Omont, A., et al. 2007, AJ, 134, 2435

Wilson, T. L., \& Batrla, W. 2005, A\&A, 430, 561

Woosley, S. E., \& Weaver, T. A. 1995, ApJS, 101, 181

Yan, L., Tacconi, L. J., Fiolet, N., et al. 2010, ApJ, 714, 100

Zhukovska, S., Gail, H., \& Trieloff, M. 2008, A\&A, 479, 453 